\title{
Microbial Diversity and Fungal Symbiont of Termite Ecosystem
}

\author{
V. Gomathi*, A. Ramalakshmi and K. Ramasamy \\ Department of Agricultural Microbiology, Tamil Nadu Agricultural University, \\ Coimbatore - 641 003, Tamil Nadu, India \\ *Corresponding author
}

\section{A B S T R A C T}

Termite mound is a rich source of nutrients and applied as a fertilizer, thus improving the productivity of soil. The role of termites in increasing the productivity of forest soils depends mainly on the micro flora presents in the gut of termites. The result of

Keywords

Termite,

Termite mound,

Fungal comb,

Termitomyces

Article Info

Accepted:

24 November 2018

Available Online:

10 December 2018 the present study revealed that the bacterial population in termite soil ranged from 68.4 - $138.2 \times 10^{6} \mathrm{cfu} / \mathrm{g}$ sample dry weight. While the fungi and actinomycetes populations ranged from $32.0-88.5 \times 10^{3} \mathrm{cfu} / \mathrm{g}$ and $12.8-28.2 \times 10^{3} \mathrm{cfu} / \mathrm{g}$ sample dry weight respectively. Among the bacterial population, Azotobacter and Beijerinckia were found predominant bacteria and both are produced higher levels of exopolysaccharide. The bacterial population of the fungus combs ranged from 25.6$36.2 \times 10^{6} \mathrm{cfu} / \mathrm{g}$ sample dry weight. The fungi and actinomycetes populations ranged from $92.8-112.8 \times 10^{4}$ and $8.4-13.1 \times 10^{3} \mathrm{cfu} / \mathrm{g}$ sample dry weight respectively. The results indicated that the fungal population was higher in fungal comb when compared to bacteria present in termite soil. The predominant fungus present in fungal comb was identified as Termitomyces. Further the Termitomyces growth condition was optimized and the study revealed that cellulose is a preferred carbon for the growth of Termitomyces compared with other tested sugars and $\mathrm{pH}$ ranged between $4.5-5.0$ and temperature between $37-40^{\circ} \mathrm{C}$ were identified as suitable $\mathrm{pH}$ and temperature for their effective growth of Termitomyces isolates.

\section{Introduction}

Forests provide shelter to number of soil organisms, birds and animals. Forest trees yield timber and other minor products like oils, resins and the thus contributing a part of our national income. Even at world level, forests provide a source of income to people. Forest soils are naturally rich in nutrients and many soil organisms like earth worm
(Drummond, 1987) and play as vital a role as earthworms in i) turning the soil over ii) altering the physical properties of the soil and iii) mixing organic matter with the soil and facilitating its decomposition. Termites play an important role in flow of energy and cycling of nutrients, and the economically and ecologically and important aspect of tropical ecosystem. The termite gut inhabits a variety of microflora which play an important role in 
improving the soil productivity. Aerobic, facultative aerobic, cellulose digesting, and anaerobic bacteria of gut symbionts and their digestive enzymes play a vital role in the degradation of polmers, simultaneously a fungal symbiont viz., Termitomyces help in the litter degradation of forest ecosystem.

\section{Termites and their distribution}

Termites are insects belonging to the order Isoptera. There are approximately 2000 termite species of which at least 20 are from India (Singh and Singh, 1981). In this forest ecology, termites were observed to dwell at heights of more than $30 \mathrm{~m}$ on and in the standing trees and down to a depth of $30 \mathrm{~cm}$ in the soil (Erhard, 1951b). A high degree of phylogenetic correlation exists with the geographic distribution area of the world of the late Mesozoic era and subsequent differentiation seems to have occurred locally during the tertiary times (Krishna, 1970).

The principal ecological factors that influence the dispersal of termites in India are the monsoon rainfall pattern (Mean annual rainfall, mean number of rain days), atmospheric temperature, atmospheric humidity, vegetation, altitude, soil type natural enemies and other associated organisms, Termites Like Microctermes and Nasutitermes and Macrotermes are characteristics of forest vegetation. Most rain forests have relatively large number of genera of termites.

\section{Gut microflora of termites}

The gut of termites is inhabited by diversified groups of microflora. These include bacteria, fungi, actinomycetes and protozoa. In case of bacteria, both aerobe and anaerobic bacteria are present in the gut. Aerobic bacteria like Azotobacter and Beijerinckia and facultative anaerobic bacteria like Klebsiella are nitrogenfixing bacteria which fix nitrogen, thereby enrich the soil (Erhard, 1951a). Furthermore, the nitrogen fixation by gut symbionts also plays a significant role in termite nutrition.

Termites depend on a range of microflora in their guts to facilitate digestion of the plant material. The fungi like Termitomyces help in degradation of lignocellulosic material, there by aid in organic matter decomposition. The bacteria like Azotobacter and Beijeinckia produce polysaccharides, thus help on soil aggregation. So it is clear that the termites help in improving the productivity of forest soils mainly through the microflora present in their gut. Saria and their coworkers reported that fungus comb microbiotas are largely termite species-specific due to major contributions from gut deposits and also that environment affects which gut bacteria dominate comb communities at a given point in time.

\section{Improvement in soil physical properties}

The termites help in turning over the soil and they influence the pattern of horizonation. It has been reported that the rate of soil being transferred to the deep horizon every year by termite activity was about $0.025 \mathrm{~mm}$ (Nye, 1955). In one generation, termite mounds upto $12.5 \mathrm{~m}^{3} /$ ha of soil i.e. $20 \mathrm{t} / \mathrm{ha}$. This implied that the $20 \mathrm{~cm}$ thick A-horizon may have taken about 8000 years to accumulate. Termites are, therefore, an important soil forming factor in the tropics. The role of termites in the morpho genetic processes or profile development of soils has long been recognized. The activity of a mound builder resulted in a complete mixing up of the surface layer 25 to $35 \mathrm{~cm}$ deep. Even the deep horizons were found to be pierced by tunnels and chambers upto $25 \mathrm{~m}$ from the centre of a termitorium. Termites affect the soil structure in many ways. The indirect effects of termite of termite activist on soil structure are through the rapid mineralization of crop residue and 
organic wastes. Termite galleries are often back - filled and later occupied by plant rots that enrich them with organic matter and stabilize them (Lee and Wood, 1968). These channels and feeding galleries created in a termite - infested soil may increase infiltration and water transmission (Harns, 1965).

The soil - water retention properties of termite infested soil depend on the alternation created by their activity in soil's pore - size distribution. In India, more soil moisture equivalent and maximum water-holding capacity was observed in the samples of Termitorium built by Hypotermesobscuripes (Table 1) as compared to those of adjacent soils (Pathak and Lehrilk, 1956). The increase in water retention was obviously due to high organic matter content. The higher clay and lower sand silk content of the termite pockets was responsible for nitrogen proportion of water retention pores. Termites are found to help in controlling soil erosion. The positive effects of termite activity in reducing erosion are due to high infiltration of water through the channels and feeding galleries. If these crusts occur beneath the soil surface, they also conserve soil - water in the root above the nested horizons. In most regions, soils of the mound are found to be more fertile and more productive (Pendletion, 1941; Mielke, 1978). Mound soils are traditionally used as fertilizer and a source of lime (Rounce, 1949). Hence termite soils provide favourable environment for crop growth (Table 2).

\section{Organic matter formation and $\mathrm{N}_{2}$ fixation}

More organic matter was present in termitorium than in surrounding soils, which is probably due to the use of body fluids and excretion mound construction and to the accumulation of biomass as food reserves. The wood feeding termites generally has a higher $\mathrm{C}$ : $\mathrm{N}$ ration than grass feeders (Lee and Wood, 1968). This is because termites can live on a diet of cellulose and there may be a possibility of nitrogen fixation in termites. It was observed that nitrification and nitrogen fixation in the soil of a termite nest was higher than that in the control (Pathak and Lehrilk, 1956). A higher level of microbiological activities was observed in termite nest than in normal soil.

The termite mounds of Macrotermes sp. contain more fertile materials than surrounding soil because they contain more cellulose decomposers, denitrifiers, ammonifiers and nitrifiers than the surrounding soil (Meikkehon, 1965). The quantitative data on nirtrigen fixation by different termites has also been reported (Breznak et al., 1973). The $\mathrm{C}_{2} \mathrm{H}_{4}$ reducing ability of remitters was studied. All castes of termites reduced $\mathrm{C}_{2} \mathrm{H}_{4}$ possessed the lowest activity. There were also differences among species regarding their ability to reduce $\mathrm{CO}_{2}$. Both the aerobes (Azotobacter, Beijerinckia) and facultative anaerobes (Klebsiella, Clostridioum) were found to fix atmospheric nitrogen.

\section{Termites in soil aggregation}

Most termites use soil together with saliva and feces to construct their nests. Greater water holding capacity and cation exchange capacity of termite - infested soils are primarily due to their higher contents of clay and organic matter (Sheik and Kayain, 1982). The nutrient status of mound was usually better than that of the surrounding top soils (Pathak and Lehrilk, 1956). A soil aggregate has been defined as a naturally occurring cluster of group of soil particles in which the forces holding the particles together are much stronger than the forces between adjacent aggregation (Martin et al., 1955). A role for microbial products in soil aggregate formation leads to the study in the development of synthetic and natural conditioners (Page, 1983). In an attempt to 
increase the productivity of the soil, much attention has been focused on soil aggregation and stabilization (9.15). A soil crumb is a soil aggregate composed primarily of soil particles and binding agents such as microbial cell, microbial products and organic matter.

In acid lateritic soils, termite construct nests and in doing so, they form stable soil crumbs, with the help of microbes and their products. Such crumbs help in receiving and retaining more rainwater and increasing soil biological processes, ultimately improving the crop yield. The stability of soil aggregate is directly related to the microbial; decomposition of the added organic material. The duration of the aggregation effect and the time taken to reach the maximum depend on the nature of the substrate.

\section{Factors affecting soil aggregation}

Environmental factors play a major role in stabilizing the soil aggregates. Addition of organic matter to the soil affect the $\mathrm{pH}$ condition of the soil which is unfavourable for the microbial growth, thereby restrict the aggregate stabilization. The 9growth of bacterial population of polysaccharide producers was observed. Gomathi and Ramasamy (1991) also reported that temperature $381^{\prime} \mathrm{C}$ favour more microbial growth, which are involved in stable soil aggregates.

\section{Microbes \& Microbial products in aggregation}

Microbial population in soil aggregate was enumerated and compared with control. It was found that more predominant bacteria were Azotobacte rand Beijerinckia. Fungi were also present but very less in number. Totally, actinomycetes was nil in the aggregate sample. It is well know that bacterial polysaccharides and polyuronides are generally present in soils in sufficient concentration and are particularly effective in enhancing aggregation (Renney et al., 1954). A positive relationship was found between the acid content of bacterial polymers and their stability effect in case of Azotobacter (Gaur and Rao, 1975). Large quantities of gums were produced by A.chroococcum consists or $87 \%$ glucose and $3 \%$ uronic acid (Copper et al., 1938) Galacturonic acid and gulcuronic acid have been shown to play an important role in the stabilization of soil aggregates (Gaur and Rao, 1975). Both Azotobacter and Beijericnkia produced higher, levels of exopolysaccharide and the results are presented in Table 3.

\section{Microbial population in Termite soil}

The highest bacterial population was recorded in Mettupalayam sample and the highest fungal and actinomycetes population were recorded in the samples from Ooty and kodaikanal respectively. There was only a slight difference between locations. The bacterial population in termite soil ranged from 68.4 - $138.2 * 10^{6} \mathrm{cfu} / \mathrm{g}$ sample dry weight. While the fungi and actinomycetes populations ranged from $32.0-88.5^{*} 10^{3} \mathrm{cfu} / \mathrm{g}$ and $12.8-28.2 * 10^{3} \mathrm{cfu} / \mathrm{g}$ sample dry weight respectively. The isolated bacteria were identified as Bacillus, Pseudomonas, Azotobacter, Beijerinckia, Arthrobacter and Serratia. Fungi were identified based on morphological characteristics as Trichoderma,

Fusarium, Rhizopus, Aspergillus and Actinomycetes as Streptomyces.

\section{Microbial population in fungus combs}

The bacterial population of the fungus combs ranged from $25.6-36.2 \times 10^{6} \mathrm{cfu} / \mathrm{g}$ sample dry weight. The fungi and actinomycetes populations ranged from $92.8-112.8 \times 10^{4}$ and $8.4-13.1 \times 10^{3} \mathrm{cfu} / \mathrm{g}$ sample dry weight respectively. 
Table.1 Biological activity of termite nest soils (Hypotermes obscuripes)

\begin{tabular}{|l|l|l|}
\hline Biological activity & Termite nest & Control \\
\hline Nitrogen fixation (g) & 0.350 & 0.011 \\
\hline Nitrification (ppm) & 1064.000 & 140.000 \\
\hline $\mathrm{CO}_{2}$ evolution (mg \% after 24 hours) & 31.700 & 10.100 \\
\hline Total N after incubation & 1.520 & 0.061 \\
\hline N fixed per g pf C oxidized & 0.140 & 0.013 \\
\hline
\end{tabular}

Table.2 $\mathrm{N}_{2}\left(\mathrm{C}_{2} \mathrm{H}_{2}\right)$ fixation in termites

\begin{tabular}{|c|c|c|c|c|}
\hline \multirow[t]{2}{*}{ Termite } & \multirow[t]{2}{*}{ Caste } & \multirow[t]{2}{*}{ Diet } & \multicolumn{2}{|c|}{$\mathrm{C}_{2} \mathrm{H}_{2}$ (n mol formed per $h$ ) } \\
\hline & & & $\begin{array}{l}\text { per } g \\
\text { termites }\end{array}$ & $\begin{array}{l}\text { per } g \\
\text { termites }\end{array}$ \\
\hline $\begin{array}{l}\text { Coptoermes } \\
\text { formosanus }\end{array}$ & $\begin{array}{l}\text { Worker } \\
\text { Soldier }\end{array}$ & $\begin{array}{l}\text { Wood } \\
\text { Wood }\end{array}$ & $\begin{array}{l}0.122 \\
0.027\end{array}$ & $\begin{array}{l}0.695 \\
0.139\end{array}$ \\
\hline Reticulitermes flacipes & $\begin{array}{l}\text { Worker } \\
\text { Soldier }\end{array}$ & $\begin{array}{l}\text { Wood } \\
\text { Wood }\end{array}$ & $\begin{array}{l}0.043 \\
0.014\end{array}$ & $\begin{array}{l}0.204 \\
0.073\end{array}$ \\
\hline Zootermopsis sp. & $\begin{array}{l}\text { Reproductive } \\
\text { nymohs \& } \\
\text { workers }\end{array}$ & Wood & 0.517 & 0.272 \\
\hline Cryptotermes brevis & $\begin{array}{l}\text { Reproductive } \\
\text { nymphs }\end{array}$ & $\begin{array}{l}\text { Moist filter } \\
\text { paper }\end{array}$ & 0.622 & 1.705 \\
\hline
\end{tabular}

(Brezank et al., 1973)

Table.3 Production of polysaccharide and its component sugars

\begin{tabular}{|c|c|c|c|}
\hline Organism & & mg/g of carbon & Component sugars \\
\hline A.chroococcum & & 603 & R,.M.,F,.G, MN \\
\hline A.vinelandii & & 820 & M.,G,GL \\
\hline Beijerinckia 1 & & 813 & I.,M.,F,.GI \\
\hline Beijerinckia 2 & & 820 & $\mathrm{I}, . \mathrm{F}, \mathrm{M}, . \mathrm{Mn}, \mathrm{GL}$ \\
\hline Beijerinckia 3 & & 640 & I.,F,.Mn,.GL \\
\hline $\begin{array}{l}\mathrm{R} \text { - Rhamnose } \\
\mathrm{M} \text { - Mannose }\end{array}$ & $\begin{array}{l}\text { G - Glucose } \\
\text { I - Inositol }\end{array}$ & $\begin{array}{l}\text { G1 - Galactose } \\
\text { F - Fructose }\end{array}$ & n - Mannitol \\
\hline
\end{tabular}


Table.4 Biomass production of Termitomyces in different carbon sources

\begin{tabular}{|c|c|c|c|}
\hline \multirow{2}{*}{ Isolates } & \multicolumn{3}{|c|}{ Biomass (g/100 $\mathrm{ml}$ of broth) } \\
\hline & $\begin{array}{l}\text { Carboxy methyl } \\
\text { cellulose }\end{array}$ & Starch & Xylose \\
\hline $\mathbf{T m}_{1}$ & $2.78( \pm 0.15)^{\mathrm{c}}$ & $2.95( \pm 0.18)^{b}$ & $3.05( \pm 0.05)^{\mathrm{ab}}$ \\
\hline $\mathrm{Tm}_{2}$ & $2.95( \pm 0.02)^{b}$ & $2.55( \pm 0.15)^{\mathrm{c}}$ & $3.55( \pm 0.02)^{\mathrm{a}}$ \\
\hline $\mathrm{Tm}_{3}$ & $3.01( \pm 0.05)^{\mathrm{a}}$ & $3.10( \pm 0.20)^{\mathrm{a}}$ & $2.90( \pm 0.15)^{\mathrm{c}}$ \\
\hline $\mathrm{Tm}_{4}$ & $2.15( \pm 0.02)^{\mathrm{c}}$ & $3.10( \pm 0.20)^{\mathrm{a}}$ & $2.95( \pm 0.50)^{\mathrm{c}}$ \\
\hline $\mathrm{Tm}_{5}$ & $2.90( \pm 0.05)^{b}$ & $2.20( \pm 0.15)^{\mathrm{cd}}$ & $3.25( \pm 0.03)^{b}$ \\
\hline $\mathrm{Tm}_{6}$ & $2.75( \pm 0.01)^{\mathrm{c}}$ & $2.45( \pm 0.25)^{\mathrm{c}}$ & $3.12( \pm 0.06)^{\mathrm{ab}}$ \\
\hline $\mathbf{T m}_{7}$ & $3.00( \pm 0.02)^{\mathrm{a}}$ & $2.90( \pm 0.05)^{\mathrm{b}}$ & $2.95( \pm 0.02)^{\mathrm{c}}$ \\
\hline $\mathrm{Tm}_{8}$ & $2.45( \pm 0.03)^{\mathrm{bc}}$ & $1.01( \pm 0.02)^{\mathrm{h}}$ & $1.85( \pm 0.15)^{\mathrm{e}}$ \\
\hline $\mathbf{T m}_{9}$ & $2.10( \pm 0.05)^{\mathrm{cd}}$ & $2.05( \pm 0.20)^{\mathrm{def}}$ & $2.06( \pm 0.05)^{\mathrm{bc}}$ \\
\hline $\mathrm{Tm}_{10}$ & $1.98( \pm 0.15)^{\mathrm{e}}$ & $1.32( \pm 0.25)^{\mathrm{efg}}$ & $1.90( \pm 0.05)^{\mathrm{cd}}$ \\
\hline $\mathrm{Tm}_{11}$ & $1.65( \pm 0.25)^{\mathrm{ef}}$ & $1.75( \pm 0.03)^{\mathrm{ef}}$ & $2.15( \pm 0.45)^{\mathrm{abc}}$ \\
\hline $\mathrm{Tm}_{12}$ & $1.75( \pm 0.25)^{\mathrm{ef}}$ & $2.15( \pm 0.05)^{\mathrm{e}}$ & $1.75( \pm 0.15)^{\mathrm{cd}}$ \\
\hline $\mathrm{Tm}_{13}$ & $2.10( \pm 0.15)^{\mathrm{cd}}$ & $2.00( \pm 0.21)^{\mathrm{def}}$ & $1.95( \pm 0.12)^{\mathrm{cd}}$ \\
\hline $\mathrm{Tm}_{14}$ & $2.01( \pm 0.05)^{\mathrm{de}}$ & $2.05( \pm 0.05)^{\mathrm{def}}$ & $2.06( \pm 0.02)^{b c}$ \\
\hline $\mathrm{Tm}_{15}$ & $2.32( \pm 0.02)^{b c}$ & $1.12( \pm 0.25)$ & $1.56( \pm 0.03)^{\mathrm{ef}}$ \\
\hline $\mathrm{Tm}_{16}$ & $2.05( \pm 0.05)^{\mathrm{d}}$ & $1.85( \pm 0.03)^{\mathrm{tg}}$ & $1.35( \pm 0.15)$ \\
\hline $\mathbf{T}_{17}$ & $1.85( \pm 0.15)^{\mathrm{ef}}$ & $1.90( \pm 0.05)^{\mathrm{fg}}$ & $2.05( \pm 0.25)^{\mathrm{bc}}$ \\
\hline$T_{18}$ & $1.92( \pm 0.02)^{\mathrm{e}}$ & $2.15( \pm 0.20)^{\mathrm{e}}$ & $2.09( \pm 0.05)^{\mathrm{bc}}$ \\
\hline$T_{19}$ & $1.75( \pm 0.15)^{\mathrm{ef}}$ & $1.95( \pm 0.05)^{\mathrm{fg}}$ & $2.10( \pm 0.03)^{\mathrm{bc}}$ \\
\hline$T_{20}$ & $2.05( \pm 0.15)^{d}$ & $1.65( \pm 0.03)^{\mathrm{efg}}$ & $2.15( \pm 0.16)^{\mathrm{abc}}$ \\
\hline
\end{tabular}

Values are mean $\pm \operatorname{SEd}(n=3)$. Means followed by a common letter are not significantly different to each other according to DMRT $(p \leq 0.05)$. 
Int.J.Curr.Microbiol.App.Sci (2018) 7(12): 3283-3295

Table.5 Effect of $\mathrm{pH}$ on growth of Termitomyces

\begin{tabular}{|c|c|c|c|c|c|}
\hline \multirow{2}{*}{ Isolates } & \multicolumn{5}{|c|}{ Growth (cm) } \\
\hline & 4.5 & 5.5 & 6 & 7 & 8 \\
\hline $\mathbf{T m}_{1}$ & $2.50( \pm 0.05)^{\mathrm{a}}$ & $4.40( \pm 0.18)^{\mathrm{e}}$ & $2.05( \pm 0.05)^{b c}$ & $1.10( \pm 0.03)^{\mathrm{ab}}$ & $0.40( \pm 0.03)^{\mathrm{b}}$ \\
\hline $\mathrm{Tm}_{2}$ & $2.00( \pm 0.02)^{\mathrm{cd}}$ & $4.50( \pm 0.15)^{\mathrm{de}}$ & $1.80( \pm 0.02)^{\mathrm{d}}$ & $1.00( \pm 0.12)^{b}$ & $0.30( \pm 0.02)^{\mathrm{bc}}$ \\
\hline $\mathbf{T m}_{3}$ & $2.30( \pm 0.05)^{\mathrm{b}}$ & $5.00( \pm 0.20)^{\mathrm{c}}$ & $2.00( \pm 0.15)^{\mathrm{bc}}$ & $0.90( \pm 0.03)^{\mathrm{c}}$ & $0.10( \pm 0.01)^{\mathrm{e}}$ \\
\hline $\mathrm{Tm}_{4}$ & $2.00( \pm 0.02)^{\mathrm{cd}}$ & $5.10( \pm 0.20)^{\mathrm{abc}}$ & $1.80( \pm 0.50)^{\mathrm{d}}$ & $0.80( \pm 0.05)^{\mathrm{de}}$ & $0.50( \pm 0.10)^{\mathrm{a}}$ \\
\hline $\mathrm{Tm}_{5}$ & $2.50( \pm 0.05)^{\mathrm{a}}$ & $5.20( \pm 0.15)^{\mathrm{ab}}$ & $2.00( \pm 0.03)^{b c}$ & $0.98( \pm 0.04)^{b c}$ & $0.35( \pm 0.03)^{\mathrm{abc}}$ \\
\hline $\mathrm{Tm}_{6}$ & $2.01( \pm 0.01)^{\mathrm{c}}$ & $4.85( \pm 0.25)^{\mathrm{cde}}$ & $1.95( \pm 0.06)^{\mathrm{cd}}$ & $0.88( \pm 0.02)^{\mathrm{cd}}$ & $0.35( \pm 0.02)^{\mathrm{abc}}$ \\
\hline $\mathbf{T m}_{7}$ & $2.20( \pm 0.02)^{b}$ & $4.90( \pm 0.05)^{\mathrm{de}}$ & $2.02( \pm 0.02)^{\mathrm{c}}$ & $0.85( \pm 0.04)^{\mathrm{cd}}$ & $0.45( \pm 0.05)^{\mathrm{ab}}$ \\
\hline $\mathrm{Tm}_{8}$ & $2.25( \pm 0.03)^{\mathrm{ab}}$ & $5.01( \pm 0.02)^{\mathrm{c}}$ & $1.85( \pm 0.15)^{\mathrm{d}}$ & $0.95( \pm 0.05)^{b c}$ & $0.50( \pm 0.03)^{\mathrm{a}}$ \\
\hline $\mathbf{T m}_{9}$ & $2.10( \pm 0.05)^{\mathrm{abc}}$ & $5.25( \pm 0.20)^{\mathrm{ab}}$ & $2.06( \pm 0.05)^{b}$ & $0.75( \pm 0.12)^{\mathrm{e}}$ & $0.30( \pm 0.02)^{\mathrm{bc}}$ \\
\hline $\mathrm{Tm}_{10}$ & $1.98( \pm 0.15)^{\mathrm{d}}$ & $5.32( \pm 0.25)^{\mathrm{a}}$ & $1.90( \pm 0.05)^{\mathrm{c}}$ & $0.80( \pm 0.02)^{\mathrm{de}}$ & $0.25( \pm 0.03)^{\mathrm{cd}}$ \\
\hline $\mathbf{T m}_{11}$ & $1.65( \pm 0.25)^{\mathrm{e}}$ & $5.25( \pm 0.03)^{\mathrm{b}}$ & $2.15( \pm 0.45)^{\mathrm{a}}$ & $1.01( \pm 0.10)^{\mathrm{ab}}$ & $0.54( \pm 0.02)^{\mathrm{a}}$ \\
\hline $\mathrm{Tm}_{12}$ & $1.75( \pm 0.25)^{\mathrm{cd}}$ & $4.95( \pm 0.05)^{\mathrm{cd}}$ & $1.75( \pm 0.15)^{\mathrm{d}}$ & $0.89( \pm 0.03)^{\mathrm{d}}$ & $0.25( \pm 0.10)^{\mathrm{cd}}$ \\
\hline $\mathrm{Tm}_{13}$ & $2.00( \pm 0.15)^{\mathrm{cd}}$ & $5.00( \pm 0.21)^{\mathrm{c}}$ & $1.95( \pm 0.12)^{\mathrm{cd}}$ & $0.76( \pm 0.02)^{\mathrm{e}}$ & $0.20( \pm 0.02)^{\mathrm{de}}$ \\
\hline $\mathrm{Tm}_{14}$ & $2.01( \pm 0.05)^{\mathrm{c}}$ & $4.65( \pm 0.05)^{\mathrm{e}}$ & $2.06( \pm 0.02)^{b}$ & $0.95( \pm 0.15)^{\mathrm{bc}}$ & $0.31( \pm 0.03)^{\mathrm{bc}}$ \\
\hline $\mathrm{Tm}_{15}$ & $2.32( \pm 0.02)^{b}$ & $5.02( \pm 0.25)^{\mathrm{cd}}$ & $1.56( \pm 0.03)^{\mathrm{de}}$ & $0.80( \pm 0.25)^{\mathrm{de}}$ & $0.20( \pm 0.01)^{\mathrm{de}}$ \\
\hline $\mathrm{Tm}_{16}$ & $2.05( \pm 0.05)^{b c}$ & $4.85( \pm 0.03)^{\mathrm{de}}$ & $1.35( \pm 0.15)^{\mathrm{e}}$ & $1.01( \pm 0.14)^{\mathrm{ab}}$ & $0.15( \pm 0.02)^{\mathrm{de}}$ \\
\hline$T_{17}$ & $1.85( \pm 0.15)^{\mathrm{de}}$ & $4.90( \pm 0.05)^{\mathrm{d}}$ & $2.05( \pm 0.25)^{\mathrm{abc}}$ & $1.15( \pm 0.05)^{\mathrm{a}}$ & $0.19( \pm 0.13)^{\text {cde }}$ \\
\hline$T_{18}$ & $1.92( \pm 0.02)^{\mathrm{d}}$ & $5.25( \pm 0.20)^{b}$ & $2.09( \pm 0.05)^{b}$ & $0.98( \pm 0.02)^{b c}$ & $0.32( \pm 0.12)^{b c}$ \\
\hline$T_{19}$ & $1.75( \pm 0.15)^{\mathrm{de}}$ & $4.85( \pm 0.05)^{\mathrm{de}}$ & $2.10( \pm 0.03)^{\mathrm{ab}}$ & $1.12( \pm 0.02)^{\mathrm{a}}$ & $0.26( \pm 0.03)^{\mathrm{cd}}$ \\
\hline$T_{20}$ & $2.05( \pm 0.15)^{\mathrm{bc}}$ & $4.65( \pm 0.03)^{\mathrm{e}}$ & $2.15( \pm 0.16)^{\mathrm{a}}$ & $0.90( \pm 0.03)^{\mathrm{cd}}$ & $0.34( \pm 0.02)^{\mathrm{c}}$ \\
\hline
\end{tabular}

Values are mean $\pm \operatorname{SEd}(n=3)$. Means followed by a common letter are not significantly different to each other according to DMRT $(p \leq 0.05)$. 
Int.J.Curr.Microbiol.App.Sci (2018) 7(12): 3283-3295

Table.6 Effect of temperature on growth of Termitomyces

\begin{tabular}{|c|c|c|c|c|c|c|}
\hline \multirow[t]{2}{*}{ Isolates } & \multicolumn{5}{|c|}{ Growth $(\mathrm{cm})$} & \multirow[b]{2}{*}{50} \\
\hline & 0 & 15 & 27 & 37 & 42 & \\
\hline $\mathrm{Tm}_{1}$ & $0.10( \pm 0.01)^{\mathrm{c}}$ & $\begin{array}{c}0.90 \\
( \pm 0.03)^{\mathrm{de}}\end{array}$ & $\begin{array}{c}5.00 \\
( \pm 0.20)^{\mathrm{abc}}\end{array}$ & $1.10( \pm 0.03)^{\mathrm{a}}$ & $0.54( \pm 0.02)^{\mathrm{a}}$ & $\begin{array}{c}0.14 \\
( \pm 0.02)^{\mathrm{abc}}\end{array}$ \\
\hline $\mathrm{Tm}_{2}$ & $0.0( \pm 0.0)^{\mathrm{e}}$ & $0.80( \pm 0.05)^{\mathrm{e}}$ & $\begin{array}{c}5.10 \\
( \pm 0.20)^{\mathrm{ab}}\end{array}$ & $1.00( \pm 0.12)^{b}$ & $\begin{array}{c}0.25 \\
( \pm 0.10)^{\mathrm{de}}\end{array}$ & $\begin{array}{c}0.15 \\
( \pm 0.03)^{\mathrm{ab}}\end{array}$ \\
\hline $\mathrm{Tm}_{3}$ & $0.16( \pm 0.02)^{b}$ & $\begin{array}{c}0.98 \\
( \pm 0.04)^{\mathrm{de}}\end{array}$ & $5.20( \pm 0.15)^{\mathrm{a}}$ & $\begin{array}{c}0.90 \\
( \pm 0.03)^{\mathrm{abc}}\end{array}$ & $\begin{array}{c}0.20 \\
( \pm 0.02)^{\mathrm{de}}\end{array}$ & $\begin{array}{c}0.14 \\
( \pm 0.02)^{\mathrm{abc}}\end{array}$ \\
\hline $\mathrm{Tm}_{4}$ & $0.15( \pm 0.01)^{b}$ & $0.88( \pm 0.02)^{\mathrm{e}}$ & $\begin{array}{c}4.85 \\
( \pm 0.25)^{\mathrm{cd}}\end{array}$ & $0.80( \pm 0.05)^{\mathrm{de}}$ & $\begin{array}{c}0.31 \\
( \pm 0.03)^{\mathrm{cd}}\end{array}$ & $\begin{array}{c}0.12 \\
( \pm 0.01)^{\mathrm{bc}}\end{array}$ \\
\hline $\mathrm{Tm}_{5}$ & $0.0( \pm 0.0)^{\mathrm{e}}$ & $\begin{array}{c}1.98 \\
( \pm 0.15)^{\mathrm{ab}}\end{array}$ & $5.25( \pm 0.20)^{\mathrm{a}}$ & $0.98( \pm 0.04)^{\mathrm{bc}}$ & $\begin{array}{c}0.20 \\
( \pm 0.01)^{\mathrm{de}}\end{array}$ & $0.0( \pm 0.0)^{\mathrm{e}}$ \\
\hline $\mathrm{Tm}_{6}$ & $0.10( \pm 0.01)^{\mathrm{c}}$ & $\begin{array}{c}1.65 \\
( \pm 0.25)^{\mathrm{cd}}\end{array}$ & $5.32( \pm 0.25)^{\mathrm{a}}$ & $0.88( \pm 0.02)^{\mathrm{cd}}$ & $\begin{array}{c}0.15 \\
( \pm 0.02)^{\text {cde }}\end{array}$ & $\begin{array}{c}0.10 \\
( \pm 0.01)^{\mathrm{de}}\end{array}$ \\
\hline $\mathrm{Tm}_{7}$ & $0.22( \pm 0.02)^{\mathrm{a}}$ & $\begin{array}{c}1.75 \\
( \pm 0.25)^{\mathrm{cd}}\end{array}$ & $5.25( \pm 0.03)^{\mathrm{a}}$ & $0.85( \pm 0.04)^{\mathrm{cd}}$ & $\begin{array}{c}0.19 \\
( \pm 0.13)^{\text {cde }}\end{array}$ & $0.22( \pm 0.02)^{c}$ \\
\hline $\mathrm{Tm}_{8}$ & $0.20( \pm 0.03)^{\mathrm{a}}$ & $\begin{array}{c}1.75 \\
( \pm 0.15)^{\mathrm{cd}}\end{array}$ & $\begin{array}{c}4.40( \pm \\
0.18)^{\mathrm{e}}\end{array}$ & $0.95( \pm 0.05)^{b c}$ & $\begin{array}{c}0.32 \\
( \pm 0.12)^{b c}\end{array}$ & $0.20( \pm 0.03)$ \\
\hline $\mathrm{Tm}_{9}$ & $\begin{array}{c}0.15 \\
( \pm 0.01)^{\mathrm{ab}}\end{array}$ & $\begin{array}{c}1.95 \\
( \pm 0.12)^{\mathrm{abc}}\end{array}$ & $\begin{array}{c}4.50 \\
( \pm 0.15)^{\mathrm{de}}\end{array}$ & $0.75( \pm 0.12)^{\mathrm{e}}$ & $\begin{array}{c}0.26 \\
( \pm 0.03)^{\mathrm{cd}}\end{array}$ & $\begin{array}{c}0.15 \\
( \pm 0.01)^{\mathrm{ab}}\end{array}$ \\
\hline $\mathrm{Tm}_{10}$ & $0.0( \pm 0.0)^{\mathrm{e}}$ & $2.06( \pm 0.02)^{\mathrm{a}}$ & $\begin{array}{c}4.85 \\
( \pm 0.03)^{\mathrm{cd}}\end{array}$ & $0.80( \pm 0.02)^{\mathrm{de}}$ & $\begin{array}{c}0.34 \\
( \pm 0.02)^{\mathrm{bc}}\end{array}$ & $\begin{array}{c}0.10 \\
( \pm 0.01)^{\mathrm{cd}}\end{array}$ \\
\hline $\mathrm{Tm}_{11}$ & $0.0( \pm 0.0)^{\mathrm{e}}$ & $\begin{array}{c}1.56 \\
( \pm 0.03)^{\mathrm{cd}}\end{array}$ & $4.90( \pm 0.05)^{\mathrm{c}}$ & $1.01( \pm 0.10)^{\mathrm{b}}$ & $\begin{array}{c}0.40 \\
( \pm 0.03)^{\mathrm{ab}}\end{array}$ & $0.0( \pm 0.0)^{\mathrm{e}}$ \\
\hline $\mathrm{Tm}_{12}$ & $0.0( \pm 0.0)^{\mathrm{e}}$ & $\begin{array}{c}1.35 \\
( \pm 0.15)^{\text {de }}\end{array}$ & $5.25( \pm 0.20)^{\mathrm{a}}$ & $0.89( \pm 0.03)^{\mathrm{cd}}$ & $\begin{array}{c}0.30 \\
( \pm 0.02)^{\mathrm{cd}}\end{array}$ & $\begin{array}{c}0.16 \\
( \pm 0.02)^{\mathrm{ab}}\end{array}$ \\
\hline $\mathbf{T m}_{13}$ & $\begin{array}{c}0.03 \\
( \pm 0.01)^{\mathrm{de}}\end{array}$ & $1.85( \pm 0.15)^{\mathrm{c}}$ & $\begin{array}{c}4.85 \\
( \pm 0.05)^{\mathrm{cd}}\end{array}$ & $0.76( \pm 0.02)^{\mathrm{de}}$ & $0.10( \pm 0.01)^{\mathrm{e}}$ & $\begin{array}{c}0.15 \\
( \pm 0.01)^{\mathrm{ab}}\end{array}$ \\
\hline $\mathrm{Tm}_{14}$ & $0.05( \pm 0.01)^{\mathrm{d}}$ & $\begin{array}{c}1.92 \\
( \pm 0.02)^{\mathrm{bc}}\end{array}$ & $\begin{array}{c}4.65 \\
( \pm 0.03)^{\mathrm{de}}\end{array}$ & $0.95( \pm 0.15)^{\mathrm{bc}}$ & $0.50( \pm 0.10)^{\mathrm{a}}$ & $0.0( \pm 0.0)^{\mathrm{e}}$ \\
\hline $\mathrm{Tm}_{15}$ & $\begin{array}{c}0.15 \\
( \pm 0.03)^{\mathrm{ab}}\end{array}$ & $\begin{array}{c}1.75 \\
( \pm 0.15)^{\mathrm{cd}}\end{array}$ & $\begin{array}{c}4.95 \\
( \pm 0.05)^{\mathrm{cd}}\end{array}$ & $0.80( \pm 0.25)^{\mathrm{e}}$ & $\begin{array}{c}0.35 \\
( \pm 0.03)^{\text {bc }}\end{array}$ & $\begin{array}{c}0.15 \\
( \pm 0.01)^{\mathrm{ab}}\end{array}$ \\
\hline $\mathrm{Tm}_{16}$ & $0.03( \pm 0.01)^{\mathrm{e}}$ & $2.05( \pm 0.15)^{\mathrm{a}}$ & $\begin{array}{c}5.00 \\
( \pm 0.21)^{\mathrm{abc}}\end{array}$ & $1.01( \pm 0.14)^{\mathrm{b}}$ & $\begin{array}{c}0.35 \\
( \pm 0.02)^{\mathrm{bc}}\end{array}$ & $0.0( \pm 0.0)^{\mathrm{e}}$ \\
\hline $\mathbf{T}_{17}$ & $\begin{array}{c}0.14 \\
( \pm 0.02)^{\mathrm{abc}}\end{array}$ & $\begin{array}{c}1.95 \\
( \pm 0.06)^{\mathrm{abc}}\end{array}$ & $4.65( \pm 0.05)$ & $1.15( \pm 0.05)^{\mathrm{a}}$ & $\begin{array}{c}0.45 \\
( \pm 0.05)^{\mathrm{ab}}\end{array}$ & $0.0( \pm 0.0)^{\mathrm{e}}$ \\
\hline $\mathrm{T}_{18}$ & $0.15( \pm 0.03)^{\mathrm{ab}}$ & $\begin{array}{c}2.02 \\
( \pm 0.02)^{\mathrm{ab}}\end{array}$ & $5.02( \pm 0.25)^{b c}$ & $0.98( \pm 0.02)^{b c}$ & $0.50( \pm 0.03)^{\mathrm{a}}$ & $0.0( \pm 0.0)^{\mathrm{e}}$ \\
\hline $\mathrm{T}_{19}$ & $\begin{array}{c}0.14 \\
( \pm 0.02)^{\mathrm{abc}}\end{array}$ & $1.85( \pm 0.15)^{\mathrm{c}}$ & $\begin{array}{c}5.01 \\
( \pm 0.02)^{\mathrm{bc}}\end{array}$ & $1.12( \pm 0.02)^{\mathrm{a}}$ & $\begin{array}{c}0.30 \\
( \pm 0.02)^{\mathrm{cd}}\end{array}$ & $\begin{array}{c}0.03 \\
( \pm 0.01)^{\mathrm{de}}\end{array}$ \\
\hline$T_{20}$ & $\begin{array}{c}0.12 \\
( \pm 0.01)^{\mathrm{bc}}\end{array}$ & $2.06( \pm 0.05)^{\mathrm{a}}$ & $4.90( \pm 0.05)$ & $\begin{array}{c}0.90 \\
( \pm 0.03)^{\mathrm{abc}}\end{array}$ & $\begin{array}{c}0.25 \\
( \pm 0.03)^{\mathrm{abc}}\end{array}$ & $\begin{array}{c}0.05 \\
( \pm 0.01)^{\mathrm{de}}\end{array}$ \\
\hline
\end{tabular}

Values are mean $\pm \operatorname{SEd}(n=3)$. Means followed by a common letter are not significantly different to each other according to DMRT $(p \leq 0.05)$. 
The results indicated that the fungal population was higher when compared to bacteria and fungi. Among six locations, the Thadiyankudisai sample recorded the highest microbial population. The isolated organisms were identified as Bacillus, Azotobacter, Beijerinckia and Pseudomonas. Fungi were identified based on morplological characteristics as Termitomyces, Aspergillus, Penicillium, Trichoderma, Fusarium, Xylaria and the actinomycetes as streptomyces.

\section{Termites and fungi}

Termites play a significant role in the decomposition of plant residue and vegetation biomass. The fungi in the gut of termites and in degradation of lignocellulosic wastes. Heim (1940) placed all the agents that develop from termitorium under the genus Termitomyces. The termites usually change the lignin cellulose ration in the plant debris using this fungus. It was reported that Nasutitermes exitious changed the lignin to cellulose ratio from 1:5 in their food (dead wood) to 5:1 in their mounds (Lee and Wood, 1971). The termites tend to favour the mineralization of plant debris rather than its humification (Baschlier, 1977).

Termites of subfamily Macrotermitinae, so called fungus growing termites have sophisticated and highly efficient symbiotic relationship with fungi. Fungus growing termites are abundant in Asia and African tropics have a great impact on the decomposition of dead plant material in those ecosystem. In the nest of the termites, the symbiotic fungi grow on a sponge like structure (called a fungus comb) constructed by the termites from a litter. They are found as mycelia and white round structures (called fungus nodules) on the fungus comb surface. The termites consume both the fungi and fungus comb. Termitomyces mushroom appears on the termite nests in a particular season. The mushrooms are unique in nature, blooming only from the termite nests, and are commercially fascinating due to their prized edibility. Termitomyces mushroom has long pseudo rhiza which connects to the surface of the fungus comb. Termitomyces grown in a special modified media General soil fungus Media, novobiacin as antibacterial agent. The cultures grown under in vitro condition at temperature of $30+{ }^{0} \mathrm{C}$

Effects of different carbon sources on the growth of Termitomyces isolates under in vitro conditions

The different carbon sources like glucose, xylose, cellulose and starch were used for the growth of Termitomyces cultures for their utilization pattern. In potato dextrose broth, xylose, cellulose and starch were used as carbon sources at the concentration of $1 \%$ instead of glucose. The Termitomyces cultures were inoculated and incubated under controlled condition of $32-37 \mathrm{C}$ after 3 weeks of incubation the growth intensity of Termitomyces was recorded in all the four carbon sources and the results indicated that the Termitomytes cultures grow very well in cellulose followed by glucose, starch and xylose. The study revealed that cellulose is a preferred carbon for the growth of Termitomyces compared with other tested sugars (Table 4).

\section{Standardization of substrate concentration for the growth of Termitomyces isolates}

Different substrates viz., saw dust, coir waste, maize cob and paddy straw were used for its growth study. The substrate were sterilized and the extracts were collected, the collected extracts were added in PDA medium in different concentrations viz., 5\%, 10\%, 15\% and $20 \%$. The Termitomyces isolates were inculated in all the concentration of the substrate and incubated. The observation was 
made after 15 days of inoculation of Termitomyces isolates. The best growth of Termitomyces was recorded in 5 and $10 \%$ of saw dust and maize cob.

\section{Standardization of $\mathrm{pH}$ for the growth of Termitomyces isolates under invitro condition}

Potato dextrose broth was prepared with varying $\mathrm{pH}$ levels viz., 4.5, 5.5, 6.0, 7.0 and 8.0. Termitomyces fungal isolates were inoculated and the broth was kept under controlled condition. The growth of Termitomyces cultures was recorded after 3 weeks of inoculation (Table 5). The $\mathrm{pH}$ ranged between $4.5-5.0$ was identified as suitable $\mathrm{pH}$ for their effective growth.

\section{Standardization of incubation temperature for the growth of Termitomyces Isolates under invitro condition}

The termitomyces cultures ware inoculated in potato dextrose broth and incubated under different temperatures viz., $0^{0} \mathrm{C} .15^{0} \mathrm{C}, 27^{0} \mathrm{C}$, $37^{\circ} \mathrm{C}, 42^{\circ} \mathrm{C}$ and $50^{\circ} \mathrm{C}$ and kept under controlled condition for their effective growth.

Of Termitomyces was recorded after 3 weeks (Table 6). The results showed that $37-40^{\circ} \mathrm{C}$ temperature was found to be optimum for the growth of termitomyces isolates.

The symbiotic fungi have been proposed to play a ligninolytic role to improve digestibility of cellulose for the termites, to supply cellulase and xylanase which work synergistically with endogenous enzymes and to concentrate nutrients, particularly nitrogen for the termites (Taprab et al., 2002).

Termites constitute an important group of soli animals and play a major role in the forest ecosystem. Termites help in horizonation of soil and development of soil profile, thus help in improving the soil forming processes. Termite mound is a rich source of nutrients and can be applied as fertilizer, thus improving the productivity of soil. The role of termities in increasing the productivity of forest soils depends mainly on the microflora presents in the gut of termites. Aerobic like Azotobacter and Beijerinckia and facultative anaerobic bacteria like Klebsiella and clostridium fix atmospheric nitrogen and contribution to soil nitrogen. Celluloytic and lignin degrading fungi help in degradation of lignocellulosic waste, resulting in mineralization and these wastes could be used as compost to increase the productivity of the soil. The soil aggregates formed by polysaccharide producing bacteria Azotobacter and Beijernckia increased the water retention properties of the soil. It also increase rooting moisture and prevents soil erosion. The soil crumbs formed by polysaccharide producing bacteria increases rooting moisture uptake by plants. Thus the important beneficial effects of termites (due to involvement of gut micro flora are i) soil turn over ii) addition of plant nutrients and recycling iii) alteration in soil physical properties i.e. texture iv) improvement in soil porosity and v) decomposition of plant litter (Termite symbiotic fungi viz., Termitomyces). Thus it is clear that gut microflora of termites help in improving the yield of forest products, thereby paving a way for increasing national income.

\section{Termites (white ants)}

Major contributors to the breakdown of organic matter at or near surface

\section{2,000 species}

Found in $2 / 3$ of the land surface area but most prominent in grasslands or savannas and forest of tropical and subtropical areas 
Up to 16 billion in one hectare in tropical deciduous forest

In drier areas, termites surpass earthworms in domination the soil fauna

\section{Termite Mounds}

Social organism building mounds of cemented soil, prominent in Africa, Latin America, Australia and Asia

Form cities of networks that may extend 10 to 20 meters beyond the mound

Consume mostly rotting wood and materials and plant residues

Some African species use OM to grow fungi on mounds as major source of food

Mix soil by bringing soil from subsurface areas to build mound moving from 300 to $1200 \mathrm{~kg} / \mathrm{ha}$ soil to build mounds

Remove up to $4000 \mathrm{Kg} / \mathrm{ha}$ of leaf and woody material annually

Mound of 6 meters or higher consisting of 2.4 $\mathrm{kg} / \mathrm{ha}$

Mounds are the homes of 1 million or more individuals

An existing mound is difficult to knock downs they rebuild very rapidly

\section{Effect of termites on soil productivity}

Localize organic matter into mound while denuding adjacent areas making it difficult to keep Om residues on soils

More efficient in breakdown of organic matter with gut microbes
Growth Around Mounds

Less nutrient rich because they build from sub soils

If subsoil is richer than topsoil or of clay instead of the sandy soil usually found in topsoil, then may provide islands of enriched soils with phosphorus, potassium, calcium and moisture

Increase water infiltration into the soil in subtropical and tropical can tropical areas where surface is crusted and penetration is difficult

Increase decay of dead trees and grasses

Disrupt crop production, road construction, because of rapid mound building Bacterial metabolism

\section{References}

Baschlier, G.C (1977). Review of action of termites in soil. Science du soil, 1: 3-12.

Breznak, J.A., W.J, J.W. Mertins and H.C. Coppel. (1973). Nitrogen fixation in termites. Nature, 244: 577-580.

Copper, E.A., W.D. Daker and M. Stacey. (1938). Enzyme formation and polysaccharide synthesis by bacteria. Pat III: polysaccharides produced by nitrogen fixing organisms. Biochem. J. 32:17521758.

Drummond, H. (1987). Termite as the tropical analogue of the earthworm. Proc. R. Soc. (E.dinb) 13:13, 14.

Erhard, H. (1951a). The importance of biological phenomena in the formation of ferruginous must in the tropic. C. R. Acad. Sci (Paris). 233: 804-806.

Erhard, H. (1951b). The role of termite nests in the geography of tropical regions C.R. Academic Sci (Paris), 233:966-96. 
Gaur, A.C., and R.V.S. Rao. (1975). Note on the isolation of bacterial gums and their influence on soil aggregate stabilization. Ind. J. Agr. Sci., 45: 86-89.

Gomathi, V. and K. Ramasamy. (1991). Isolation and characterization of microbial products involved in stable soil aggregation. ICARAshoc. Project, TNAU, Coimbatore-3

Haris R.F., G. Cheaters and Allen. (1966). Dynamics of soil aggregation. Adv. Agron., 18: 107-169.

Harns, W.V (1965). Termites and the soil. Soil zool. proc. Nottingham sch. Agric. Sci., 62-72.

Heim, R. (1940). Les champignonnieres des termites les grands champignons d; AfriueTropicale. Rev. Bot. Appl., 20:121127.

Krishna, K. (1970). Taxonomy, phylogeny and distribution of termities In: Biology of termities vol. II Academic press, New York and London. pp. 127-152.

Lee K.F. and T.G. Wood (1971). Physical and chemical effects on soil of some Australian termities and their pedological significance, pedobiologia, 16: 451-456.

Lee, K. F. and T.G. Wood. (1968) Preliminary studies of the role of nasuitermesexitiosus in the cycling of organic mateer in a yellow podzolic soil under dry sclephll forest in south Australia-Trans. $9^{\text {th }}$ Int. Cong. Soil., 2:1-18.

Lynch, J.M. and Bragg E (1985). Microorganisms and aggregate stability. Adv. Soil. Sci., 2: 133-170.

Martin, J.P., W.P. Martin, J, B. Page, W.A. Raney and J.G. Demert. (1955). Soil aggregation. Adv. Agron., 7: 1-37.

Meikkehon, J. (1965). Microbiological studies on large termite mounds- Rhos. Zambia Malaaawi. J. Agri. Res., 3: 67-79.

Mielke H.W. (1978). Termitoria and shifting cultivation, The dynamic role of the termite insoils of tropical wet dry Africa, Trop. Ecol. 19: 117-122.
Noparatnaraporn, N. and T. Kudo. (2002). Molecular phylogeny of symbiotic basidiomycetes of fungus growing termites is Thailand and their relationship worth the host. Biosci. Biotechnol. Biochem. 66(5): 11591163.

Nye P.H. (1955). Some soil forming processes in the humizaltropice, iv. The action of the soil fauna. J. Soil. Sci., 6: 73-83.

Page E.R. (1983). Forest biomes in soil cnditiones. Span, 26: 10-11.

Pathak A. N. and Lehrilk (1956). Studies on termite nests chemical physical and biological characteristic of a termitiorium in relation to tis surroundings. J. Ind. Soc. Soil. Sci., 7: 87-90.

Pendletion R. L. (1941). Some results of termite activity in Thailand Soils, Thai Sci. Bull., 3: 29-53.

Pomeroy, D. E. (1983). Some effects of mound abuilding termites on soils of semi-arid area Kenya. J. soil Sci., 34: 555-570.

Quinnell, C. M., S. G. Knight and P. W. Wilson (1957). The polysaccharide produced by Azotobcterindicum. Can. J. Microbiol., 3: 277-288.

Renney, D. A., E. Triog and Allen (1954). Soil aggregation as influenced by micribial level of fertility and kind of crip. Soil. soc. Amer. Proc., 18: 399 403.

Roonwal M. L. and O.B. Chhotani. (1996). Revision of the termite genus Enztermes (Termided: Amitermitinae) Proc. Naty. Sci. India, 31: 81-113.

Rounce N.V. (1949). Manuring in its relation to land rehabilitation. In: The Agriculture of the cultivation steppe (Rounce Nved.) Longmans Green \& Co, London, pp. 25-35. 
Sheik, K. H. and A. S. Kayain. (1982). Termite affected soils in Pakistan. Soil Biol. Biochem., 14: 359-364.

Singh U. R. and J.S. Singh. (1981). Populatyion, structure and mound architecture of the termites of a tropical deciduous frost of Varanasi, India, Pedobiologia 22: 213-223.
Taprab. Y.M. Ohkuma., T. Johjima., Y. Maeds., S. Moriya., T. Inouse., P. Suwanarit., N. Noparatnarporn and T. Kudo. (2002). Molecular phylogeny of symbiotic basidionychnol. Biochem. 66 (5): 1159-1163.

\section{How to cite this article:}

Gomathi, V., A. Ramalakshmi and Ramasamy, K. 2018. Microbial Diversity and Fungal Symbiont of Termite Ecosystem. Int.J.Curr.Microbiol.App.Sci. 7(12): 3283-3295.

doi: https://doi.org/10.20546/ijcmas.2018.712.380 